\title{
A Phase II Study of Prophylactic Non-Irradiated Granulocyte Transfusions in AML Patients Receiving Induction Chemotherapy
}

\author{
Fleur Aung ${ }^{1^{*}}$, Courtney D DiNardo ${ }^{2}$, Fernando Martinez ${ }^{1}$, Sherry Pierce ${ }^{2}$, Naval Daver ${ }^{2}$, Tapan Kadia ${ }^{2}$, Elias Jabbour ${ }^{2}$, Hagop Kantarjian $^{2}$, Benjamin Lichtiger ${ }^{1}$ \\ and Emil J Freireich ${ }^{2}$ \\ ${ }^{1}$ Department of Laboratory Medicine, University of Texas, MD Anderson Cancer Center, Houston, TX, USA \\ ${ }^{2}$ Department of Leukemia, University of Texas, MD Anderson Cancer Center, Houston, TX, USA
}

"Corresponding author: Fleur M. Aung, Department of Laboratory Medicine, University of Texas, MD Anderson Cancer Center, Houston, TX, USA, Tel: 713-792-8630; Fax: 713-792-2634; E-mail: fmaung@mdanderson.org

Received date: Sep 10, 2016, Accepted date: Feb 3, 2017, Publication date: February 9, 2017

Copyright: (c) 2017 Aung F, et al. This is an open-access article distributed under the terms of the Creative Commons Attribution License, which permits unrestricted use, distribution, and reproduction in any medium, provided the original author and source are credited.

\begin{abstract}
Background: Patients with Acute Myeloid Leukemia (AML) experience profound neutropenia; infections remain the leading cause of morbidity and mortality. Transfusion of functional non-irradiated allogeneic granulocytes may treat or prevent infections in AML patients, and may also have anti-leukemic benefits.
\end{abstract}

Study Design: Patients free of infection, with a diagnosis of AML or high-risk myelodysplastic syndrome undergoing induction or first-salvage therapy were eligible. Allogeneic Granulocyte Transfusions (GTs) were administered to neutropenic $\left(<0.5 \times 10^{9} / \mathrm{L}\right)$ patients every 3-4 days until sustained ANC recovery, initiation of new therapy, or completion of 6 weeks on study.

Results: 45 patients enrolled with a median age of 67 years (range 23-83); 27 (60\%) were male. Five patients (11\%) never received a GT, due to donor screening failure and/or donor unavailability. 119 donors donated 156 granulocyte concentrates to the remaining 40 patients. The median number of GTs transfused per patient was 3 (range 1-9). All patients experienced $>1$ neutropenic fever, with an average of one infectious episode per patient. Other adverse reactions were urticaria/pruritis $(n=1)$, rash $(n=1)$, and hypotension $(n=1)$. Response to leukemiadirected therapy included complete remission in $50 \%$, overall response rate of $70 \%$, and 8 -week mortality of $8 \%$. Median overall survival was 15 months, with $51 \% 1$-year survival.

Conclusion: Administration of non-irradiated functional allogeneic GTs to neutropenic MDS/AML patients is safe and feasible. No transfusion-associated graft-versus-host-disease (TA-GVHD) was reported and no increased toxicity was described, including among the $10 \%$ receiving subsequent allogeneic stem cell transplant. The favorable patient outcomes within this diverse group of primarily elderly AML are notable.

Keywords: Granulocyte transfusion; AML; Neutropenia; Prophylaxis

\section{Introduction}

Patients with acute myeloid leukemia (AML) experience profound and sustained neutropenia during induction chemotherapy. The current standard of care is to provide neutropenic patients with broadspectrum antibiotic, antifungal and antiviral therapy while their absolute neutrophil count is suppressed $[1,2]$. However, resistance to available anti-infective agents is increasing and even with this standard approach many patients still succumb to neutropenic infections [3-5].

Since the development of plasmapheresis technology and transfusion medicine in the 1950s [6,7], spontaneous hemorrhage as a primary cause of mortality from treatment-related myelosuppression has decreased, and infectious complications remain the leading cause of morbidity and mortality in children and adults with acute leukemia [8-10]. The degree and duration of neutropenia are predictive, with high-risk patients considered to be those with prolonged ( $>7$ days duration) and profound (absolute neutrophil count (ANC) $<100$ cells $/ \mathrm{mm}^{3}$ ) neutropenia [11].
Granulocyte transfusions (GT) to combat neutropenic infections were pioneered in the 1960's. Early success was limited by two main factors; red blood cell contamination and poor granulocyte yield. As white blood cell (WBC) density is similar to red blood cell (RBC) density, GTs obtained during pheresis demonstrated heavy RBC contamination. And with only $\sim 5 \%$ of total body neutrophils circulating in the peripheral circulation at a given time, the number of recovered WBCs was minimal $\left(\sim 1 \times 10^{10}\right)$, the majority were lymphocytes, and a median increment in ANC of only $\sim 100 / \mu$ l was observed per GT. Technology has since improved, including strategies to combat RBC contamination such as the addition of the nonimmunogenic hydroxyethyl starch (HES) to cause RBC rouleaux phenomenon and improve the efficiency of granulocyte separation, as well as the development of the continuous-flow blood cell separator [12]. In addition, routine GCSF and/or corticosteroid administration stimulate the margination of neutrophils leading to a routine obtainment of an effective granulocyte dose $[13,14]$.

Prior studies have demonstrated that in the setting of active neutropenic infections, granulocytes should be infused daily for a minimum of 4-8 days for optimal clinical effectiveness [15-17]. Granulocyte transfusions have also been utilized in the prevention/ 
prophylaxis setting for immunosuppressed leukemia patients, where responses have been mixed. A Cochrane Review in 2009 of 10 randomized prospective trials failed to demonstrate clinical benefit; notably the majority of included studies featured inadequate PMN doses $\left(<10^{10}\right)$ per transfusion; and in the seven studies with adequate granulocytes transfused, mortality was reduced by $60 \%$ [18]

Starting in the 1990s, all blood products are irradiated in an effort to eradicate donor lymphocytes and reduce the possibility of transfusionassociated acute graft versus host disease (TA-GVHD). Yet irradiation of WBC products is known to significantly diminish neutrophil and mononuclear cell function $[19,20]$. A recent randomized clinical trial of irradiated $v s$ non-irradiated GTs in adult immunosuppressed relapsed/refractory leukemia patients with refractory infections, statistically designed to demonstrate non-inferiority, determined that non-irradiated GTs had no increased toxicity, no detriment to overall survival, and did not affect the ability to proceed to stem cell transplant (SCT) or impact post-SCT outcomes. Notably, non-irradiated GTs led to significantly increased mean and median granulocyte increments per single GT [21]. With over 600 granulocyte transfusions administered, there was no reported incidence of TA-GVHD.

Herein we report the results of a Phase II study to determine (1) the feasibility of utilizing non-irradiated granulocyte transfusions as prophylactic therapy in patients with AML or high-risk MDS undergoing induction or first-salvage induction therapy and (2) to assess the preliminary efficacy of prophylactic non-irradiated granulocyte transfusions on outcome and prevention of infections in this population.

\section{Patients and Methods}

\section{Patient eligibility}

All patients were enrolled at The University of Texas MD Anderson Cancer Center following institutional guidelines. This clinical study was registered with ClinicalTrials.gov (NCT01932710). Eligibility included adult patients with AML or high-risk myelodysplastic syndrome (MDS) receiving initial induction chemotherapy, or first salvage re-induction treatment. Patients were required to be free of infections, including signs and symptoms of infection at the time of entering the study. All patients provided written informed consent.

\section{Treatment and follow-up}

Patients on study were followed with complete blood counts $(\mathrm{CBC})$ daily. In patients with an ANC <500/ $\mu$ l, GTs were administered approximately every 3-4 days based on donor availability, so long as patients remained neutropenic with an ANC $<500 / \mu l$, until (1) patients attained neutrophil recovery, (2) a period of 6-wks had elapsed, or (3) the patient transitioned to alternate therapy. Triple antimicrobial prophylaxis was employed following institutional guidelines, with oral fluroquinolone, azole or echinocandin antifungal, and antiviral therapy recommended as first-line prophylaxis and modified based on patient tolerance and drug allergy profile.

\section{Granulocyte collection}

GT donors were recruited from friends and family of leukemia patients. All potential donors met standard blood donor criteria established by current AABB (Advancing Transfusion and Cellular Therapies Worldwide) and the US Food and Drug Administration (FDA) guidelines. Written informed consent was obtained from all eligible donors prior to granulocyte collection. Donors were not selected based on HLA or ABO blood group compatibility. All donors received $600 \mu \mathrm{g}(300 \mu \mathrm{g} \times 2)$ of subcutaneous G-CSF and/or $8 \mathrm{mg}$ of dexamethasone orally 12 hours prior to granulocyte apheresis. Granulocyte concentrates (GC) were obtained using two-arm continuous-flow apheresis (COBE Spectra/Spectra Optia, TERUMO, Lakewood, CO, USA) over 2-3 hours and collected as previously described [21]. The GCs were infused within 4-24 hours of collection and stored at room temperature prior to transfusion. All non-O Granulocytes were drained of their red cells by gravity sedimentation to leave a minimum of $<5 \mathrm{~mL}$ of red cells. Granulocyte donors who met standard blood donor criteria could donate up to 5 times in 30 days, with each collection at least $>48$ hours from the previous.

In circumstances where $>7 \times 10^{10} \mathrm{GCs}$ were obtained from a single high-yield apheresis, the GCs were split to provide GTs for $>1$ eligible patient with the majority of split-products containing $>4 \times 10^{10}$ cells [22].

\section{Response criteria and statistics}

For the primary endpoint of feasibility, the proportion of patients with sufficient donors receiving prophylactic GT therapy and the $95 \%$ confidence interval (CI) was estimated. If 20 out of 50 patients have sufficient donors to participate in the preventing study, then the $95 \%$ $\mathrm{Ci}$ for this proportion will be $(0.264,0.536)$ and the feasibility of the study was confirmed. For the secondary endpoint of efficacy, patient characteristics were summarized using medians and ranges for continuous variables and frequencies and percentages for categorical variables. Categorical variables were compared using the $\chi^{2}$ or Fisher's exact test and continuous variables using the Wilcoxon rank-sum test. All p-values are 2-sided, with statistical significance defined as a $\mathrm{p}$ value of $<0.05$. Overall survival (OS) was based on the Kaplan-Meier method, with differences compared by the log-rank test. Analyses were performed using Statistica (version 12) software.

\section{Results}

From October 2013 to August 2015, a total of 45 patients were enrolled. The median age was 67 years (range 23-83 years) and $27(60 \%)$ were male. Thirty patients $(67 \%)$ were 65 years of age or older. Clinicopathologic details of all patients are provided in (Table 1).

AML, and the remainder were admitted for front-line induction chemotherapy for AML $(n=39)$ or MDS $(n=3)$. Ten (22\%) patients were diagnosed as therapy-related AML. Treatments received are detailed in (Table 1) and are classified as high-dose cytarabine-based chemotherapy $(\mathrm{n}=11 ; 24 \%)$, hypomethylating-agent based therapy $(\mathrm{n}=27 ; 60 \%)$, or low-dose cytarabine in combination with either cladribine or clofarabine $(n=7 ; 16 \%)$. Based on the induction AML prognostic model for overall survival in elderly patients developed in 2006 by Kantarjian et al. [5], 9(20\%) of patients were low-risk, corresponding to a median OS of 18 months and 8-week mortality of $10 \%$. Thirty-one (69\%) of patients were intermediate-risk, corresponding to a median OS of 7 months and a 8-week mortality of $26 \%$, and $5(11 \%)$ were high-risk, corresponding to a median OS of 1 month and $57 \%$ 8-week mortality. 
Citation: $\quad$ Aung F, DiNardo CD, Martinez F, Sherry P, Daver N, et al. (2017) A Phase II Study of Prophylactic Non-Irradiated Granulocyte Transfusions in AML Patients Receiving Induction Chemotherapy. J Blood Disord Transfus 8: 376. doi:10.4172/2155-9864.1000376

Page 3 of 10

\begin{tabular}{|c|c|}
\hline & $\mathrm{N}=45$ \\
\hline Age & 67 [range 23-83] \\
\hline Age $>65$ years & $30(67 \%)$ \\
\hline Male/Female & $27 / 18$ \\
\hline \multirow[t]{3}{*}{ Diagnosis } & 39 (87) AML Induction \\
\hline & $3(7 \%)$ AML Salvage \\
\hline & $3(7 \%)$ MDS \\
\hline Therapy-Related AML & 10 (4 MDS, 1 MPN, 3 solid tumor, 1 CLL, 1 AA) \\
\hline \multirow[t]{3}{*}{ Treatment Regimen } & $11(24 \%)$ high or intermediate dose cytarabine based \\
\hline & $27(60 \%)$ HMA based \\
\hline & $7(16 \%)$ LDAC based \\
\hline \multirow[t]{3}{*}{ Cytogenetics } & $18(40 \%)$ diploid \\
\hline & 15 (33\%) other /intermediate risk \\
\hline & $12(27 \%)$ complex /chrom 5/7 abnl \\
\hline MDACC Prognostic Model ${ }^{1}$ & $9(20 \%)$ low risk (0 factors) \\
\hline \multirow[t]{2}{*}{ (age $>75$, PS $>2$, complex cytogenetics, PE, AHD, Cr>1.3) } & $31(69 \%)$ intermediate risk (1-2 factors) \\
\hline & $5(11 \%)$ high risk (> 3 factors) \\
\hline ANC at Start Induction & 760 median [range $0-49.8 \mathrm{~K}$ ] \\
\hline PLT at Start Induction & 37 median [range $5-308 \mathrm{~K}$ ] \\
\hline
\end{tabular}

Table 1: Patient Demographics.

Three patients (7\%) were enrolled at the time of salvage reinduction chemotherapy for five of the 45 enrolled patients $(11 \%)$ never received a granulocyte transfusion, due to donor screening failure and/or donor unavailability.

These patients are thus not evaluated in either the safety or efficacy analysis, and are considered a failure with regards to the feasibility analysis. Forty patients received at least one GT and are evaluable for feasibility, safety and efficacy.

A total of 119 granulocyte donors, 40 (34\%) females and 79 (66\%) males, median age of 40 years (range 17-77) donated 156 GCs, which were transfused to 40 patients. $24(20 \%)$ of the 119 donors were repeat donors who underwent a median of 2 (range of 2-6) of Granulocytapheresis. Of the 156 Granulocytes transfused, 70 (45\%) were $\mathrm{ABO}$ matched, $38(24 \%)$ were $\mathrm{ABO}$ compatible $(\mathrm{O}$ ? A/B/AB) and the remainder 48 (31\%) were $\mathrm{ABO}$ incompatible (A?B, A?O, B? A, B?O, $\mathrm{AB}$ ?O).

All non-ABO Group "O" Granulocyte units were drained of their red cells by gravity sedimentation. All Granulocyte units were cross matched and units transfused were noted as $A B O$ matched, $A B O$ compatible or ABO incompatible. Of the $156 \mathrm{GTs}, 130(83 \%)$ were split products with a median wbc count of 4.0 (range 1.4-9.0) $\times 10^{10}$ cells per unit with the majority (92\%) of the split Granulocyte units containing $>4 \times 10^{10}$ cells.
The WBC count of the 26(17\%). Unsplit Granulocyte units were a median of 6.0 (range $4.0-12) \times 10^{10}$ cells per unit. The median platelet count of the Granulocyte units transfused was 130 (range 460-425) K/uL. T

he patient responses to the GTs were median WBC 0.4 (range -6.6 to 4.9) $\mathrm{K} / \mathrm{uL}$, median ANC 0.6 (range 0.0-5.1) K/uL and median platelet count of 8 ( -28 to 59$) \mathrm{K} / \mathrm{uL}$. Although platelets were contained in the Granulocyte units, $16(40 \%)$ patients received platelets transfusions on the day of their GTs with the majority $(80 \%)$ transfused prior to the GTs.

Of the 40 patients, $16(40 \%)$ patients who received GTs were not tested for HLA alloimmunization pre- or post-GT. Of the 24 patients tested, $5(21 \%)$ showed evidence of HLA antibodies (2 patients -HLA Class I Antibodies/1 patient-HLA Class I and Class II antibodies/3 patients-HLA Class II antibodies) and $19(79 \%)$ patients were found to have no evidence of HLA alloimmunization pre-GTs.

Of the 19 patients with no evidence of HLA alloimmunization preGTs, only $10(53 \%)$ patients were tested post GT and 5(26\%) were found to be positive for HLA antibodies (2 patients with broad reactivity against both HLA Class I and HLA Class II antigens and three with reactivity against HLA Class II Antigens only) (Tables 2 and $3)$. 
Citation: Aung F, DiNardo CD, Martinez F, Sherry P, Daver N, et al. (2017) A Phase II Study of Prophylactic Non-Irradiated Granulocyte Transfusions in AML Patients Receiving Induction Chemotherapy. J Blood Disord Transfus 8: 376. doi:10.4172/2155-9864.1000376

Page 4 of 10

\begin{tabular}{|c|c|}
\hline & $\mathrm{N}=40$ \\
\hline \# GTs received [range] & $3[1-9]$ \\
\hline \multirow[t]{9}{*}{ \# GTs received per patient } & $1: 7(18 \%)$ \\
\hline & $2: 7(18 \%)$ \\
\hline & 3: $7(18 \%)$ \\
\hline & $4: 4(10 \%)$ \\
\hline & 5: $4(10 \%)$ \\
\hline & 6: $5(13 \%)$ \\
\hline & $7: 2(5 \%)$ \\
\hline & 8: $1(3 \%)$ \\
\hline & 9: $3(8 \%)$ \\
\hline \multirow[t]{2}{*}{ Time to ANC recovery $(>1 \times 10 / L)$} & 34 [23-40] \\
\hline & *25 (63\%) did not recover during study period \\
\hline \multirow[t]{2}{*}{ Time to PLT recovery (> $100 \times 10 / L)$} & 27 [22-42] \\
\hline & *21 (53\%) did not recover during study period \\
\hline \multirow[t]{7}{*}{ Fevers/Infections During Study Period } & 71 unique febrile neutropenia episodes: \\
\hline & $18(25 \%)$ pneumonia \\
\hline & $9(13 \%)$ bacteremia \\
\hline & $2(5 \%)$ UTI \\
\hline & $3(4 \%)$ other documented infection \\
\hline & $28(39 \%)$ etiology unknown \\
\hline & $11(15 \%)$ GT-related \\
\hline \multirow[t]{4}{*}{ \# Infections Reported per Subject } & 0 Infections: $2(5 \%)$ \\
\hline & 1 Infection: 20 (50\%) \\
\hline & 2 Infections: 14 (35\%) \\
\hline & $3+$ Infections: 4 (10\%) \\
\hline \multirow[t]{7}{*}{ Response to Therapy } & CR $20(50 \%)$ \\
\hline & CRp $4(10 \%)$ \\
\hline & mCR $3(8 \%)$ \\
\hline & PR $1(3 \%)$ \\
\hline & HI $1(3 \%)$ \\
\hline & NR/SD 8 (20\%) \\
\hline & ED $3(8 \%)$ \\
\hline Allogeneic SCT (\%) & $4(10 \%)$ \\
\hline 4-wk mortality (\%) & $1(3 \%)$ \\
\hline 8-wk mortality (\%) & $3(8 \%)$ \\
\hline
\end{tabular}


Citation: Aung F, DiNardo CD, Martinez F, Sherry P, Daver N, et al. (2017) A Phase II Study of Prophylactic Non-Irradiated Granulocyte Transfusions in AML Patients Receiving Induction Chemotherapy. J Blood Disord Transfus 8: 376. doi:10.4172/2155-9864.1000376

Page 5 of 10

\begin{tabular}{|l|l|}
\hline 1 -yr OS & $51 \%$ \\
\hline Median OS & 15 months \\
\hline
\end{tabular}

Table 2: Study results.

\begin{tabular}{|c|c|c|c|c|}
\hline & $N=40$ & Fail & Med-M & $\mathrm{p}$ \\
\hline \multicolumn{5}{|l|}{ Therapy } \\
\hline HMA & 24 & 10 & 14.6 & \\
\hline LD AC & 7 & 6 & 12.1 & \\
\hline $\mathrm{HD} \mathrm{AC}$ & 9 & 4 & NR & 0.39 \\
\hline \multicolumn{5}{|l|}{ MDA Model } \\
\hline Low & 7 & 2 & 17.6 & \\
\hline Intermediate & 28 & 14 & 12.1 & \\
\hline High & 5 & 4 & 3.2 & 0.03 \\
\hline \multicolumn{5}{|l|}{ Cytogenetics } \\
\hline Complex or abnormal chrom 5/7 & 10 & 7 & 12.1 & \\
\hline Diploid & 15 & 5 & 17.6 & \\
\hline Other & 15 & 8 & 5.5 & 0.12 \\
\hline \multicolumn{5}{|l|}{ Age } \\
\hline$<65$ & 12 & 5 & 16.2 & \\
\hline$>65$ & 28 & 15 & 12.1 & 0.68 \\
\hline \multicolumn{5}{|l|}{ Number of GTs } \\
\hline 2-Jan & 14 & 6 & 10.9 & \\
\hline 5-Mar & 15 & 9 & 9.8 & \\
\hline $6+$ & 11 & 5 & 17.6 & 0.27 \\
\hline \multicolumn{5}{|l|}{ Therapy-Related AML } \\
\hline Yes & 10 & 9 & 4.6 & \\
\hline No & 30 & 11 & 17.6 & 0.008 \\
\hline \multicolumn{5}{|l|}{ Disease Status } \\
\hline Induction & 37 & 17 & 14.6 & \\
\hline Salvage & 3 & 3 & 2.3 & 0.03 \\
\hline
\end{tabular}

Table 3: Multivariate Analysis of Prognostic Factors for overall survival.

Four patients (10\%) discontinued GT therapy prior to planned study completion after one cycle of AML therapy: due to patient request related to fevers and myalgias after the first two GT transfusions $(\mathrm{n}=1)$, and due to investigator concern related to pneumonia and concern for additional GTs leading to pulmonary complications $(n=3)$.
Three Grade 3 serious adverse events (SAEs) reported; urticaria and pruritis $(n=1)$, maculopapular rash $(n=1)$ and hypotension $(n=1)$. No Grade 4 or 5 related events were reported.

All patients experienced at least one neutropenic fever during the study period, with an average of 1 infectious episode per patient. In many cases, culture-negative fevers were temporally related to GTs, occurring 24-36 hours post-GT infusion, and $15 \%$ of neutropenic fevers occurring on study were considered GT-related. 
Citation: Aung F, DiNardo CD, Martinez F, Sherry P, Daver N, et al. (2017) A Phase II Study of Prophylactic Non-Irradiated Granulocyte Transfusions in AML Patients Receiving Induction Chemotherapy. J Blood Disord Transfus 8: 376. doi:10.4172/2155-9864.1000376

Page 6 of 10

Of 71 neutropenic febrile episodes, there were 32 documented infections including pneumonia $(n=18)$, bacteremia $(n=9)$, urinary tract infection $(n=2)$, and other documented infection $(n=3)$ (Table 4$)$.

There was one allergic transfusion reaction documented in one patient who received six GTs. No other transfusion-related reactions were reported to the blood bank suggesting the possibility that there could be underreporting of febrile episodes related to GTs.
The median time for recovery of absolute neutrophil count (ANC) $>1 \times 10^{9} / \mathrm{L}$ was 34 days (range 23-40) and the median time for platelet recovery $\left(>100 \times 10^{9} / \mathrm{L}\right)$ was 27 days (range $\left.22-42\right)$ after the first cycle of induction or re-induction chemotherapy (Table 2). Representative ANC plots from two patients are provided in (Figures 1a, 1b), who received $n=7$ and $n=5$ transfusions, respectively.

\begin{tabular}{|c|c|c|c|c|c|c|c|}
\hline & \multicolumn{3}{|c|}{ Pre-GC* transfusion } & \multicolumn{3}{|c|}{ Post-GC transfusion } & \multirow[b]{2}{*}{ Transplant } \\
\hline & HLA Ab Screen & HLA Antibodies & HLA Antibodies & HLA Ab Screen & HLA Antibodies & HLA Antibodies & \\
\hline 1 & Neg & & & Pos & $\begin{array}{l}\text { Class I and Class } \\
\text { II }\end{array}$ & $\begin{array}{l}\text { Anti-A1, A11, A2, A25, A26, A29, A30, A31, } \\
\text { A32, A33, A34, A43., A66, A68, A69, A74, } \\
\text { B*44:03, B38, B39, B57. B58, Cw15, Cw17, } \\
\text { Cw18, Cw2, Cw5, Cw6, DR11, DR12, DR13, } \\
\text { DR14, DR17, DR18, DR4, DR7, DR8, DR9, } \\
\text { DR52, DR53, DQ2, DQ7, DQ8, DQ9 }\end{array}$ & Yes \\
\hline 2 & Neg & & & Pos & $\begin{array}{l}\text { Class I and Class } \\
\text { II }\end{array}$ & A2, B44, B45, B57, B76, DR8,DRB1*04:04 & Yes \\
\hline 3 & Neg & & & Pos & Class II & $\mathrm{DQ} 7 / 8 / 9$ & No \\
\hline 4 & Neg & & & Pos & Class II & DR8 & No \\
\hline 5 & Neg & & & Pos & Class II & DR11/13/14/17/18/7/8, DQ4/5/6 & \\
\hline 6 & Pos & $\begin{array}{l}\text { Class I and Class } \\
\text { II }\end{array}$ & 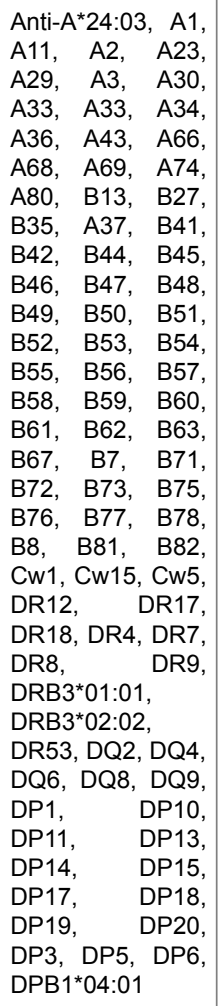 & Pos & $\begin{array}{l}\text { Class I and Class } \\
\text { II }\end{array}$ & $\begin{array}{l}\text { Anti-A*24:03, A29, A30, A33, A34, A36, A43, } \\
\text { A66, B13, B27, B35, B41, B41, B42, B44, } \\
\text { B45, B46, B47, B48, B49, B50, B51, B52, } \\
\text { B53, B54, B55, B56, B58, B59, B60, B61, } \\
\text { B62, B63, B67, B7, B71, B72, B73, B75, B76, } \\
\text { B77, B78, B81, B82, DR12, DR17, DR18, } \\
\text { DR4, DR7, DR9, DRB3*01:01, DRB3*03:01, } \\
\text { DR3, DQ2, DQ6, DQ9, DP1, DP10, DP13, } \\
\text { DP14, DP15, DP17, DP18, DP19, DP23, } \\
\text { DP28, DP3, DP5, DP6, DP9, DPB1*04:01 }\end{array}$ & No \\
\hline
\end{tabular}

Table 4: HLA Alloimmunization pre- and post-GT in 5 patients. 
Citation: $\quad$ Aung F, DiNardo CD, Martinez F, Sherry P, Daver N, et al. (2017) A Phase II Study of Prophylactic Non-Irradiated Granulocyte

Page 7 of 10

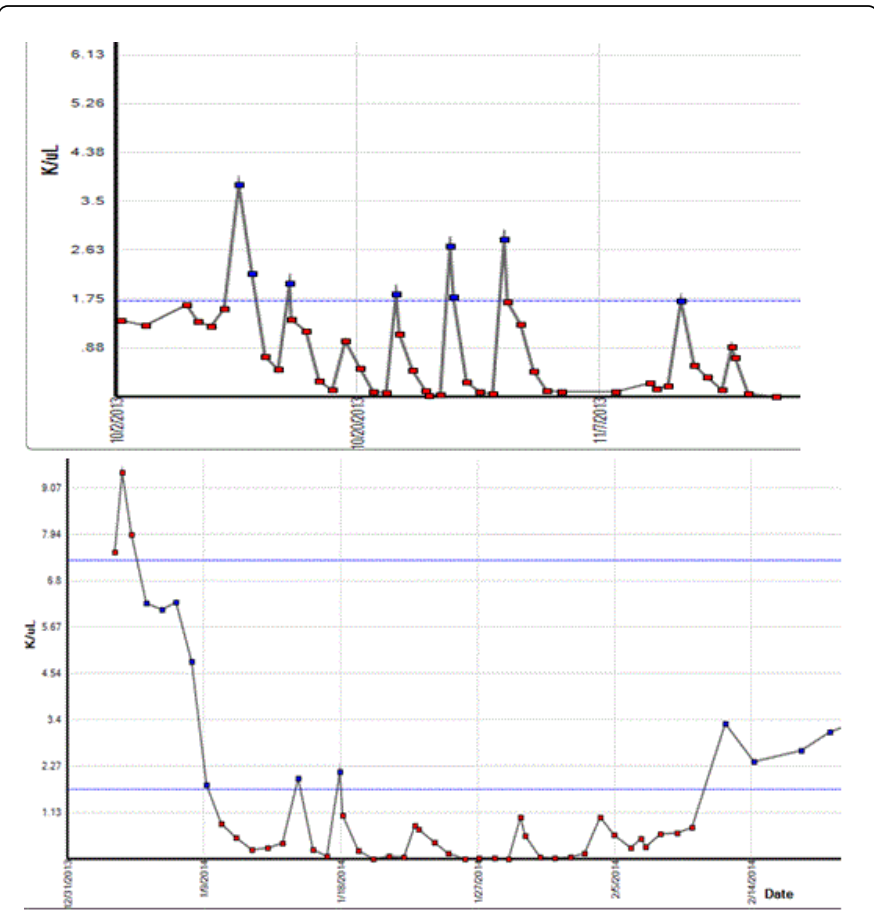

Figure 1: Representative plots of absolute neutrophil count during granulocyte transfusions.

Response to leukemia-directed therapy included a CR in $20(50 \%)$, $\mathrm{CRp}$ in $4(10 \%), \mathrm{mCR}$ in $3(8 \%), \mathrm{PR}$ in $1(3 \%)$, and hematologic improvement in $1(3 \%)$, leading to an overall response rate (ORR) of $70 \%$. No response or stable disease was observed in $8(20 \%)$ patients, and 3 patients $(8 \%)$ died within 8 -weeks of induction or reinduction therapy. Four patients went on to receive an allogeneic stem cell transplant (SCT) in remission. There was no evidence of increased SCT toxicity in these patients; notably no grade $>3$ graft-versus-host disease was documented in any patient.
With a median follow-up of 11 months, median overall survival (OS) was 15 months with a 1 -year OS of $51 \%$. The Kaplan-Meier curve of OS is provided in (Figure 2).

The MDACC prognostic model by Kantarjian et al. [5] was predictive, with a median OS of 17.6, 12.1 and 3.2 months in the low, intermediate and high-risk cohorts, respectively $(\mathrm{p}=0.03)$. Therapyrelated AML and salvage disease status were also statistically significant. Notably, the number of WBC transfusions received was not predictive of survival; a non-statistical trend towards improved median survival was noted in the patients who received 6 or more prophylactic GTs ( $\mathrm{n}=11$, with median OS $17.6 \mathrm{mo})$.

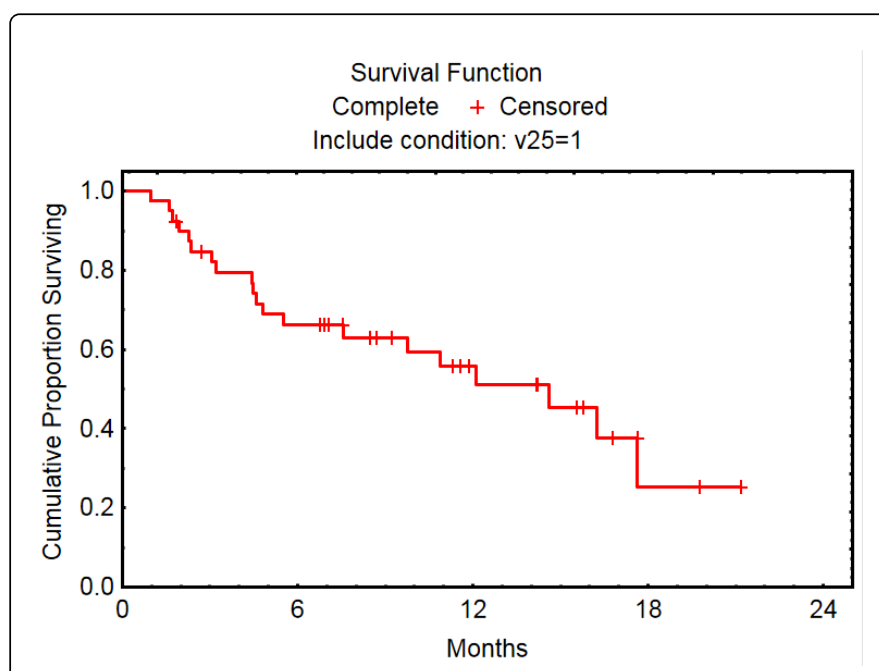

Figure 2: Overall Survival $(n=40)$ of patients on study.

A multivariate model of prognostic factors on overall survival was created and is provided in (Table 5).

\begin{tabular}{|c|c|c|c|c|c|c|c|c|c|c|c|}
\hline Patient & Gender/Age & Pt ABO & $\begin{array}{l}\text { Total GTs } \\
\text { Transfused } \\
(\mathrm{n}=156)\end{array}$ & $\begin{array}{l}\text { First } \\
\text { Degree } \\
\text { Relatives }\end{array}$ & $\begin{array}{l}\text { Second } \\
\text { Degree } \\
\text { Relative }\end{array}$ & $\begin{array}{l}\text { Not } \\
\text { Related }\end{array}$ & $\begin{array}{l}\text { ABO } \\
\text { matched } \\
\text { GTs }\end{array}$ & $\begin{array}{l}\text { ABO } \\
\text { Compatible } \\
\text { GTs }\end{array}$ & $\begin{array}{l}\text { ABO } \\
\text { Incompatible } \\
\text { GTs }\end{array}$ & $\begin{array}{lr}\text { HLA } & \text { Ab } \\
\text { Screen } & \text { Pre- } \\
\text { GTs } & \end{array}$ & $\begin{array}{lr}\text { HLA } & \text { AB } \\
\text { Screen } & \text { Post } \\
\text { GTs } & \text { (HLA } \\
\text { Ab) }\end{array}$ \\
\hline 1 & $\mathrm{M} / 65$ & $\mathrm{~A}^{+}$ & 6 & 1 & 2 & 3 & 4 & 1 & 1 & Neg & Pos(Class I /II) \\
\hline 2 & $\mathrm{M} / 71$ & A- & 9 & 3 & 4 & 2 & 6 & 3 & 0 & Not Done & Not Done \\
\hline 3 & $F / 64$ & A- & 5 & 2 & 0 & 3 & 2 & 2 & 1 & Neg & Pos (Class I/II) \\
\hline 4 & $\mathrm{~F} / 77$ & O- & 5 & 2 & 0 & 3 & 1 & 0 & 4 & Not Done & Not Done \\
\hline 5 & $\mathrm{M} / 66$ & $A+$ & 3 & 2 & 1 & 0 & 0 & 3 & 0 & Neg & Not Done \\
\hline 6 & $\mathrm{M} / 67$ & $0+$ & 3 & 1 & 1 & 0 & 3 & 0 & 0 & Neg & Pos (Class II) \\
\hline 7 & $\mathrm{M} / 67$ & O+ & 7 & 2 & 2 & 3 & 7 & 0 & 0 & Not Done & Not Done \\
\hline 8 & $F / 68$ & O+ & 4 & 0 & 0 & 4 & 0 & 0 & 4 & $\mathrm{Neg}$ & Not Done \\
\hline 9 & $\mathrm{M} / 74$ & O+ & 6 & 1 & 1 & 4 & 3 & 0 & 3 & Neg & Not Done \\
\hline 10 & $M / 64$ & $A+$ & 9 & 0 & 0 & 9 & 4 & 2 & 3 & Neg & $\mathrm{Neg}$ \\
\hline
\end{tabular}


Citation: $\quad$ Aung F, DiNardo CD, Martinez F, Sherry P, Daver N, et al. (2017) A Phase II Study of Prophylactic Non-Irradiated Granulocyte Transfusions in AML Patients Receiving Induction Chemotherapy. J Blood Disord Transfus 8: 376. doi:10.4172/2155-9864.1000376

Page 8 of 10

\begin{tabular}{|c|c|c|c|c|c|c|c|c|c|c|c|}
\hline 11 & $\mathrm{M} / 80$ & $A+$ & 2 & 0 & 0 & 2 & 0 & 1 & 1 & $\mathrm{Neg}$ & NOT Done \\
\hline 12 & $\mathrm{~F} / 78$ & O- & 3 & 0 & 0 & 7 & 4 & 0 & 3 & Not Done & Not Done \\
\hline 13 & $F / 80$ & O- & 7 & 0 & 0 & 7 & 4 & 0 & 3 & Not Done & Not Done \\
\hline 14 & $\mathrm{M} / 77$ & O+ & 9 & 2 & 3 & 4 & 5 & 0 & 4 & Not Done & Not Done \\
\hline 15 & $\mathrm{M} / 75$ & $A+$ & 8 & 0 & 1 & 7 & 2 & 6 & 0 & $\mathrm{Neg}$ & Not Done \\
\hline 16 & $F / 51$ & $A B+$ & 3 & 0 & 0 & 3 & 0 & 1 & 2 & Not Done & Not Done \\
\hline 17 & $F / 66$ & $A+$ & 3 & 0 & 2 & 1 & 1 & 0 & 2 & $\mathrm{Neg}$ & Not Done \\
\hline 18 & $F / 69$ & A+ & 2 & 0 & 0 & 2 & 1 & 1 & 0 & Neg & Neg \\
\hline 19 & $F / 68$ & $\mathrm{~A}^{+}$ & 1 & 0 & 0 & 1 & 1 & 0 & 0 & Neg & Not Done \\
\hline 20 & $\mathrm{M} / 23$ & $\mathrm{O}+$ & 1 & 0 & 0 & 1 & 1 & 0 & 0 & Not Done & Not Done \\
\hline 21 & $\mathrm{M} / 68$ & $A B+$ & 3 & 0 & 0 & 3 & 0 & 2 & 1 & Neg & Pos (Class II) \\
\hline 22 & $F / 22$ & $\mathrm{~A}^{+}$ & 2 & 0 & 1 & 1 & 0 & 2 & 0 & Pos (Class I) & Not Done \\
\hline 23 & M/59 & $A^{+}$ & 4 & 0 & 1 & 3 & 0 & 3 & 1 & Not Done & Not Done \\
\hline 24 & $F / 43$ & B- & 5 & 0 & 0 & 5 & 2 & 2 & 3 & Pos (Class I) & Not Done \\
\hline 25 & $F / 66$ & $\mathrm{~A}^{+}$ & 4 & 0 & 0 & 4 & 0 & 4 & 1 & Pos (Class I/II) & Pos (Class I/II) \\
\hline 26 & $F / 62$ & $\mathrm{O}+$ & 6 & 0 & 0 & 6 & 5 & 0 & 1 & Pos (Class II) & Not Done \\
\hline 27 & $\mathrm{M} / 79$ & $\mathrm{~A}^{+}$ & 6 & 1 & 0 & 5 & 2 & 3 & 1 & Neg & Not Done \\
\hline 28 & $\mathrm{M} / 62$ & $\mathrm{O}+$ & 1 & 0 & 0 & 1 & 1 & 0 & 0 & Not Done & Not Done \\
\hline 29 & $F / 62$ & $\mathrm{O}+$ & 3 & 0 & 0 & 3 & 3 & 0 & 0 & $\mathrm{Neg}$ & $\mathrm{Neg}$ \\
\hline 30 & $\mathrm{M} / 82$ & $\mathrm{O}+$ & 5 & 1 & 0 & 4 & 2 & 0 & 3 & Pos (Class II) & Not Done \\
\hline 31 & $\mathrm{M} / 58$ & $\mathrm{~A}^{+}$ & 6 & 0 & 0 & 6 & 2 & 4 & 0 & Neg & Neg \\
\hline 32 & $F / 77$ & $\mathrm{O}+$ & 1 & 0 & 0 & 1 & 1 & 0 & 0 & Neg & Not Done \\
\hline 33 & $\mathrm{M} / 59$ & $\mathrm{O}+$ & 2 & 0 & 0 & 2 & 2 & 0 & 0 & Neg & Pos (Class II) \\
\hline 34 & $\mathrm{M} / 69$ & O+ & 2 & 0 & 0 & 2 & 0 & 0 & 2 & Not Done & Not Done \\
\hline 35 & $\mathrm{M} / 74$ & $A B+$ & 1 & 0 & 0 & 1 & 0 & 0 & 1 & Not Done & Not Done \\
\hline 36 & $F / 66$ & O+ & 2 & 0 & 0 & 2 & 0 & 0 & 2 & Neg & Neg \\
\hline 37 & $\mathrm{M} / 63$ & O+ & 2 & 0 & 0 & 2 & 2 & 0 & 0 & Pos (Class I) & Not Done \\
\hline 38 & M/76 & O+ & 1 & 0 & 0 & 1 & 0 & 0 & 1 & Not Done & Not Done \\
\hline 39 & $F / 69$ & $\mathrm{~B}+$ & 1 & 0 & 0 & 1 & 1 & 0 & 0 & Not Done & Not Done \\
\hline 40 & $\mathrm{M} / 71$ & O+ & 3 & 0 & 0 & 3 & 1 & 0 & 2 & Not Done & Not Done \\
\hline
\end{tabular}

Table 5: Patient characteristics relating to ABO compatible GTs and HLA Alloimmunization.

\section{Discussion}

The dual objectives of this pilot study were to determine (1) the feasibility of utilizing non-irradiated GTs as prophylactic therapy in patients with AML or high-risk MDS undergoing induction or firstsalvage induction therapy, and to (2) assess the effect of prophylactically administered non-irradiated GTs on patient outcome in this high-risk population.

Feasibility was defined as the enrollment of 20 or more patients in one year with sufficient donors for GTs. Of 45 patients enrolled, 40 had sufficient donors and thus, pilot study feasibility was attained. It is nevertheless notable, that of the 40 patients with sufficient donors, 
$21(53 \%)$ received 3 or fewer GTs. In some cases, this was due to patient request to withdraw from study, or physician concern for exacerbating hypoxia in the setting of pneumonia (10\%). A priori, we anticipated approximately 8 GTs per patient, as it takes on average 4 weeks for ANC recovery during an AML treatment cycle and patients were planned to receive GTs twice per week while neutropenic. In our study, only 11 patients $(28 \%)$ received 6 or more GTs.

There was no evidence of untoward effects or toxicity related to administration of non-irradiated granulocyte transfusions. Only three significant adverse events occurred related to GT infusions, including urticaria/pruritis in one, self-resolving maculopapular rash in one, and hypotension in one. Importantly, no cases of TA-GVHD were identified despite the fact that 15 patients received 37 unirradiated GTs from first/second degree relatives.

The transfusion of HLA-mismatched peripheral blood GTs may additionally lead to an anti-leukemic effect. A recent study by Guo et al. [23] reports a significant improvement in long-term disease-free survival (DFS) in a cohort of AML patients receiving GCSF-mobilized allogeneic peripheral blood stem cells in addition to induction AML therapy, compared to induction AML therapy alone [23]. Median follow-up time on our study remains relatively short at one year; however the $51 \% 1$-yr OS and current median survival of 15 months is encouraging. Whether an improvement in long-term (i.e. 3 or 5 year) DFS will be seen in these patients will be an important future analysis, to more comprehensively evaluate for an anti-leukemic effect.

Ultimately, several barriers to routine granulocyte collections in the Blood donor center and transfusions in the clinic exist.

At the Blood Donor Center the lack and/or failure to find eligible donors preclude the patient(s) from receiving Granulocytes. Since the donors were not HLA matched, HLA alloimmunization from unirradiated granulocyte transfusions can occur and this was seen in 5 $(12.5 \%)$ of our patients. One patient showed broad reactivity to HLA Class I and Class II antigens post GTs (Table 2). This figure could possibly be higher as not all our patients were tested for HLA alloimmunization post-GTs. The risk of HLA-immunization was not prospectively addressed as a study endpoint and the data was reported in the subset of patients for whom it was available. The presence of platelets in our Granulocyte units helped and may have reduced, but did not completely eliminate the need for platelets transfusions as some of our patients had profound thrombocytopenia while undergoing induction chemotherapy. There was an effort made by the Blood Bank Physicians and supervisors to wait until the patient received the Granulocytes before dispensing platelets for transfusion. But this was not always the case as the majority (84\%) of the platelets transfused occurred earlier in the day based on the patients' counts performed earlier on that morning. Our decision to split Granulocyte units was made based solely on the needs of our patients at the time of Granulocyte collection, taking into consideration whether the Granulocyte donor was a designated donor, the volume collected and the total WBC count of the unit with the aim to provide a minimum of $4.0 \times 10^{10}$ cells per unit. It is generally believed that patients attain maximum benefit from GTs that contains the highest amounts of polymorphonuclear leucocytes (PMN). However, this clinical efficacy has not been conclusively demonstrated. A recent publication by Teofil et al. [24] suggests that patients surviving infections received standard doses $(1.5-3.0 \times 10 \mathrm{e} 8 \mathrm{cell} / \mathrm{kg})$ with a dose-related effect confirmed in bacterial infections. In the post hoc secondary analysis of the RING study, Price et al. [25] found that patients who received an average dose per transfusion of $\geq 0.6 \times 10^{9}$ granulocytes per kilogram tended to have better outcomes than those receiving a lower dose. Both studies were performed in patients with existing infections unlike our study where the primary objective was to define the feasibility of providing prophylactic granulocyte transfusion during the induction phase of treatment for AML until sustained ANC recovery, initiation of new therapy, or completion of 6 weeks on study.

In the clinic, febrile reactions are frequent and can lead to prolonged hospitalization, escalation to broad-spectrum antibiotics, and invasive procedures such as bronchoscopy to evaluate for possible infection; particularly when used in the prophylactic setting. A further difficulty with routine administration of GTs includes the notably short half-life of 12-24 hours post-collection, limiting the ability to store or transport granulocyte units. This compares to a half-life of 120 days for RBCs, and 8-10 days for platelets. An important alternative to donorcollected GTs are thus ex vivo expanded neutrophils as a manufactured product, produced from multiple donors and cryopreserved to provide an "off the shelf" myeloid progenitor product, to treat patients with prolonged neutropenia with pooled transfusions of standard quality. In these products, GCSF-mobilized peripheral or cord blood from donors is enriched for CD34+ cells and cultured in the presence of hematopoietic cytokines and/or activation of endogenous Notch signaling, leading to an expanded myeloid progenitor cell population with very low or undetectable B- and T-cell lymphoid populations [24-26]. Thus these products can transiently give rise to granulocytes and macrophages, and also retain erythroid and platelet-producing potential, however lack the ability to permanently engraft or produce lymphoid cells.

\section{Conclusion}

In conclusion, administration of non-irradiated allogeneic granulocyte transfusions to neutropenic MDS/AML patients at the time of induction therapy is feasible and safe. No evidence for TAGVHD was reported and no increased toxicity in the $10 \%$ of patients transitioned to SCT was described. Overall, patients received an average of 3 GTs on study which is detrimental to the recognition of GT-related outcomes. No significant difference on survival was identified on multivariate analysis based on the number of GTs received. The favorable ORR of $70 \%$ in a diverse group of primarily elderly AML patients receiving various treatments, along with a 1-year OS of $>50 \%$ is encouraging and warrants additional follow-up.

\section{Acknowledgment}

This work was supported in part by the MD Anderson Cancer Center Support Grant (CCSG) CA016672 and by the generous philanthropic contributions to MD Anderson's MDS/AML Moon Shot Program. CDD is also supported by the Jeanne F. Shelby Scholarship Fund which has supported her R. Lee Clark Fellow award.

\section{References}

1. Kurt B, Flynn P, Shenep JL, Pounds S, Lensing S, et al. (2008) Prophylactic antibiotics reduce morbidity due to septicemia during intensive treatment for pediatric acute myeloid leukemia. Cancer 113: 376-382.

2. Freifeld AG, Bow EJ, Sepkowitz KA, Boeckh MJ, Ito JI, et al. (2011) Clinical practice guideline for the use of antimicrobial agents in neutropenic patients with cancer: 2010 Update by the Infectious Diseases Society of America. Clin Infect Dis 52: e56-e59.

3. Carratala J, Fernandez-Sevilla A, Tubau F, Callis M, Gudiol F (1995) Emergence of quinolone-resistant Escherichia coli bacteremia in 
Citation: Aung F, DiNardo CD, Martinez F, Sherry P, Daver N, et al. (2017) A Phase II Study of Prophylactic Non-Irradiated Granulocyte Transfusions in AML Patients Receiving Induction Chemotherapy. J Blood Disord Transfus 8: 376. doi:10.4172/2155-9864.1000376

Page 10 of 10

neutropenic patients with cancer who have received prophylactic norfloxacin. Clin Infect Dis 20: 557-560.

4. Atallah E, Cortes J, O'Brien S, Pierce S, Rios MB, et al. (2007) Establishment of baseline toxicity expectations with standard frontline chemotherapy in acute myelogenous leukemia. Blood 110: 3547-3551.

5. Kantarjian H, O'brien S, Cortes J, Giles F, Faderl S, et al. (2006) Results of intensive chemotherapy in 998 patients age 65 years or older with acute myeloid leukemia or high-risk myelodysplastic syndrome: predictive prognostic models for outcome. Cancer 106: 1090-1098.

6. Hester JP, Kellogg RM, Mulzet AP, Freireich EJ (1985) Continuous-flow techniques for platelet concentrate collection: a step toward standardization and yield predictability. J Clin Apher 2: 224-230.

7. Freireich EJ (2011) Origins of platelet transfusion therapy. Transfus Med Rev 25: 252-256.

8. Bodey GP, Buckley M, Sathe YS, Freireich EJ (1966) Quantitative relationships between circulating leukocytes and infection in patients with acute leukemia. Ann Intern Med 64: 328-340.

9. Hersh EM, Bodey GP, Nies BA, Freireich EJ (1965) Causes of death in acute leukemia: a ten-year study of 414 patients from 1954-1963. JAMA 193: 105-109.

10. Rubnitz JE, Lensing S, Zhou Y, Sandlund JT, Razzouk BI, et al. (2004) Death during induction therapy and first remission of acute leukemia in childhood: the St. Jude experience. Cancer 101: 1677-1684.

11. Freifeld AG, Bow EJ, Sepkowitz KA (2011) Clinical practice guideline for the use of antimicrobial agents in neutropenic patients with cancer: 2010 Update by the Infectious Diseases Society of America. Clin Infect Dis 52: 427.

12. McCredie KB, Freireich EJ, Hester JP, Vallejos C (1974) Increased granulocyte collection with the blood cell separator and the addition of etiocholanolone and hydroxyethyl starch. Transfusion 14: 357-364.

13. Peters C, Minkov M, Matthes-Martin S, Pötschger U, Witt V, et al. (1999) Leucocyte transfusions from rhG-CSF or prednisolone stimulated donors for treatment of severe infections in immunocompromised neutropenic patients. Br J Haematol 106: 689-696.

14. Price TH, Bowden RA, Boeckh M, Bux J, Nelson K, et al. (2000) Phase I/II trial of neutrophil transfusions from donors stimulated with G-CSF and dexamethasone for treatment of patients with infections in hematopoietic stem cell transplantation. Blood 95: 3302-3309.
15. Freireich EJ (2000) Supportive care for patients with blood disorders. Br J Haematol 111: 68-77.

16. McCredie KB, Hester JP, Freireich EJ, Brittin GM, Vallejos C (1974) Platelet and leukocyte transfusions in acute leukemia. Hum Pathol 5: 699-708.

17. Clift RA, Sanders JE, Thomas ED, Williams B, Buckner CD (1978) Granulocyte transfusions for the prevention of infection in patients receiving bone-marrow transplants. N Engl J Med 298: 1052-1057.

18. Massey E, Paulus U, Doree C (2009) Granulocyte transfusions for preventing infections in patients with neutropenia or neutrophil dysfunction. Cochrane Database Syst Rev:CD0053.

19. Buescher ES, Gallin JI (1987) Effects of storage and radiation on human neutrophil function in vitro. Inflammation 11: 401-416.

20. Buescher ES, Gallin JI (1984) Radiation effects on cultured human monocytes and on monocyte-derived macrophages. Blood 63: 1402-1407.

21. Freireich EJ, Lichtiger B, Mattiuzzi G (2013) A prospective, randomized, double-blind study, comparing unirradiated to irradiated white blood cell transfusions in acute leukemia patients. Leukemia 27:861-865.

22. Aung FM, Lichtiger B, Bassett RL, Hosing C, Freireich EJ (2014) Granulocyte concentrates from a single high-yield apheresis can be split to support multiple patients. Leuk Lymphoma 55: 2577-2583.

23. Guo M, Hu KX, Yu CL, Sun QY, Qiao JH, et al. (2011) Infusion of HLAmismatched peripheral blood stem cells improves the outcome of chemotherapy for acute myeloid leukemia in elderly patients. Blood 117: 936-941.

24. Teofili L, Valentini CG, Bi Blasi R (2016) Dose-Dependent Effect of Granulocyte Transfusions in Hematologic Paitent with Febrile Neutropenia. PLos One e0159569.

25. Price TH, Boeckh M, Harrison RW, McCullough J, Ness PM, et al. (2015) Efficacy of transfusion with granulocytes from G-CSF/dexamethasonetreated donors in neutropenic patients with infection. Blood 126: 2153-2161.

26. Brunck ME, Nielsen LK (2014) Concise review: next-generation cell therapies to prevent infections in neutropenic patients. Stem Cells Transl Med 3: 541-548. 